\title{
Perfil do feminicídio: Uma abordagem epidemiológica no Estado de Sergipe
}

\author{
Profile of feminicide: An epidemiological approach in the State of Sergipe \\ Perfil del feminicidio: Un abordaje epidemiológico en el Estado de Sergipe
}

Recebido: 18/04/2021 | Revisado: 25/04/2021 | Aceito: 29/04/2021 | Publicado: 13/05/2021

Ana Clara Cruz Santos de Santana
ORCID: https://orcid.org/0000-0003-3830-0503
Universidade Federal de Sergipe Brasil
E-mail: anaclarasantana.enfa2018@gmail.com
Lucas Siqueira dos Santos
ORCID: https://orcid.org/0000-0001-5142-6931
Universidade Tiradentes, Brasil
E-mail: lucascrf648@gmail.com
Júnior de Jesus Guimarães
ORCID: https://orcid.org/0000-0002-4736-6603
Universidade Tiradentes, Brasil
E-mail: juniorguimaraes123@hotmail.com
Ikaro Daniel de Carvalho Barreto
ORCID: https://orcid.org/0000-0001-7253-806X
Universidade Federal de Pernambuco, Brasil
E-mail: daniel.carvalho.ib@gmail.com
Sonia Oliveira Lima
ORCID: https://orcid.org/0000-0002-3257-2412
Universidade de São Paulo, Brasil
E-mail: sonialima.cirurgia@gmail.com
Cláudia Moura de Melo
E-mail: franciscopradoreis@gmail.com
Universidade Tiradentes, Brasil
Oniversidade Tiradentes, Brasil
ORCID: https://orcid.org/0000-0002-7776-1831
E-mail: claudiamouramelo@hotmail.com
ORttps:/orcid.org/0000-0001-9331-003X
Orancisco Prado Reis

\begin{abstract}
Resumo
O objetivo do estudo foi estabelecer o perfil do feminicídio no estado de Sergipe no período de 2016 a 2019. Foi realizado um estudo de coorte, descritivo, exploratório, com abordagem quantitativa, por meio de coleta de dados, tendo como população mulheres acima de 18 anos, vítimas de feminicídio. No período do estudo ocorreram 262 mortes violentas de mulheres, dessas mortes 70 foram caracterizadas como feminicídios, cuja taxa de feminicídio por 100 mil mulheres variou de 1,51 a 2,61. Foram 44,3\% de feminicídios na região metropolitana e 55,7\% nos demais municípios do estado. Em todas as correlações entre as variáveis, prevaleceu como agente causador da morte o uso de armas de fogo. De igual modo, a residência prevaleceu como o local de ocorrência do feminicídio. A frequência de cor/raça parda foi maior na região metropolitana em relação aos demais municípios. Foi estatisticamente significativa a presença de mulheres economicamente ativas. Quando esta variável foi relacionada à cor/raça, esta presença foi mais expressiva entre as mulheres brancas. Quanto à escolaridade predominou, em todas as situações, a ocorrência do ensino fundamental incompleto. No Tribunal de Justiça de Sergipe, dos 31 procedimentos investigatórios instaurados, 25 foram de feminicídios, demandados por 14 das 40 Comarcas do estado de Sergipe. O feminicídio foi evidenciado neste estudo como a principal causa de violência em mulheres jovens-adultas, caracterizando dessa forma um fenômeno epidemiológico que vem se alastrando não apenas no Brasil, mas por todo o mundo.
\end{abstract}

Palavras-chave: Mulher; Homicídio; Violência contra a mulher; Vítimas de crime.

\begin{abstract}
The objective of the study was to establish the profile of feminicide in the state of Sergipe from 2016 to 2019 . A cohort, descriptive, exploratory study was carried out, with a quantitative approach, through data collection, with a population of women over 18 years, victims of femicide. During the study period there were 262 violent deaths of women, 70 of these deaths were characterized as femicides, whose rate of femicide per 100 thousand women ranged from 1.51 to 2.61. There were $44.3 \%$ of femicides in the metropolitan region and $55.7 \%$ in other municipalities in the state. In all the correlations between the variables, the use of firearms prevailed as the causative agent of death. Likewise, the residence prevailed as the place of occurrence of femicide. The frequency of color / brown race was higher in the metropolitan region in relation to the other municipalities. The presence of economically active women was statistically significant. hen this variable was related to color / race, this presence was more expressive among white women. Regarding
\end{abstract}


education, the occurrence of incomplete elementary education predominated in all situations. At the Sergipe Court of Justice, of the 31 investigative procedures initiated, 25 were of femicides, demanded by 14 of the 40 counties in the state of Sergipe. Feminicide was evidenced in this study as the main cause of violence in young adult women, thus characterizing an epidemiological phenomenon that has been spreading not only in Brazil, but throughout the world.

Keywords: Women; Murder; Violence against women; Victims of crime.

\section{Resumen}

El objetivo del estudio fue establecer el perfil del feminicidio en el estado de Sergipe en el período de 2016 a 2019. Se realizó un estudio de cohorte, descriptivo, exploratorio, con enfoque cuantitativo, a través de la recolección de datos, teniendo como población mujeres mayores de 18 años, víctimas de feminicidio. En el período del estudio, se produjeron 262 muertes violentas de mujeres, 70 de estas muertes fueron caracterizadas como feminicidios, cuya tasa de feminicidios por cada 100 mil mujeres osciló entre 1,51 y 2,61. El 44,3\% de los feminicidios se produjeron en la región metropolitana y el 55,7\% en los demás municipios del estado. En todas las correlaciones entre las variables, el uso de armas de fuego prevaleció como agente causal de la muerte. Del mismo modo, prevaleció la residencia como lugar de ocurrencia del feminicidio. La frecuencia de mestizaje fue mayor en la región metropolitana en relación con los demás municipios. La presencia de mujeres económicamente activas fue estadísticamente significativa. Cuando esta variable se relacionó con el color/raza, esta presencia fue más expresiva entre las mujeres blancas. En cuanto a la escolarización, la educación primaria incompleta predominaba en todas las situaciones. En el Tribunal de Justicia de Sergipe, de los 31 procedimientos de investigación abiertos, 25 estaban relacionados con feminicidios, demandados por 14 de los 40 distritos del estado de Sergipe. El feminicidio se evidenció en este estudio como la principal causa de violencia en mujeres jóvenes-adultas, caracterizando así un fenómeno epidemiológico que se ha extendido no sólo en Brasil, sino en todo el mundo.

Palabras clave: Mujeres; Homicidio; Violencia contra las mujeres; Víctimas de delitos.

\section{Introdução}

Em meados da década de 1970, a violência contra as mulheres no Brasil passou a ser entendida como uma questão de saúde pública. Desde este período, pesquisadores têm estudado sobre o feminicídio com o objetivo de identificar e oferecer informações sobre a morte de mulheres, resultante da discriminação, opressão, desigualdade e violência sistemática. Em termos de distribuição geográfica, a África e as Américas são as regiões em que há mais risco de as mulheres serem assassinadas por companheiros e familiares. No ano de 2017, o maior número de mulheres mortas por parceiros íntimos ou familiares foi na Ásia (20.000), seguida pela África (19.000), Américas (8.000), Europa (3.000) e Oceania (300). A África, com uma taxa de homicídio por parceiro íntimo/família de 3,1 por 100.000 habitantes femininos, é a região onde as mulheres correm maior risco de serem mortas por seus parceiros íntimos ou familiares, enquanto a Europa ( 0,7 por 100.000 habitantes) é a região onde o risco é menor (Brasil, 2016; Brasil, 2018).

Em conformidade com a Organização Mundial de Saúde (OMS) e o Relatório Mundial sobre a Prevenção da Violência, em 2014, 475 mil pessoas foram vítimas de homicídio em todo o mundo, resultando numa taxa de 6,7 mil para cada 100 mil habitantes. Em 2017, 87.000 mulheres foram mortas intencionalmente. Mais da metade delas (58\%), cerca de 50.000 vítimas, foram mortas por parceiros íntimos ou familiares. Significa que 137 mulheres em todo o mundo são mortas por um membro de sua própria família todos os dias, equivalente a seis mulheres, mortas a cada hora. De acordo com o anuário e atlas da violência, no Brasil, entre 2016 e 2019, foram registrados, em geral, 190.848 assassinatos, desse total 15.154 mil foram de mulheres, correspondendo os quatro anos à uma taxa de 16 para cada 100 mil habitantes. Nesse mesmo período ocorreram 3.904 mortes por feminicídio no Brasil com um total de taxa de 4,4 para cada 100 mil habitantes. Essa taxa de feminicídio no Brasil é a quinta maior do mundo, atrás apenas de El Salvador, Colômbia, Guatemala e da Rússia (Silva, et al., 2013; Who, 2015; Who, 2016).

Ante a esta situação e objetivando aumentar a rede de proteção ao público feminino, à vista dessa situação adversa em sociedade, foi instituída a Lei n ${ }^{\circ}$ 11.340, de 7 de agosto de 2006, popularmente conhecida como Lei Maria da Penha 10,4,5. O feminicídio tem sido imaginado por alguns autores como uma forma de genocídio, visto que está relacionado ao extermínio deliberado de um grupo social, tão somente pela condição de serem do sexo feminino. Essa atitude infracionária não pode permanecer percebida como mera discriminação, porquanto vai além das mortes cometidas por parceiros íntimos. Pode ocorrer 
após uma agressão sexual, psicológica ou qualquer outra ação ameaçadora da dignidade da mulher, seja ela cometida por pessoas conhecidas ou não. Assim, o feminicídio pode ser caracterizado como íntimo, não íntimo, e com ou sem violência sexual (Gomes, 2018; Meneghel, et al., 2017).

Dentro da temática criminal, a violência contra a mulher tem sido um assunto bastante discutido e estudado. A mulher é considerada a principal vítima da violência de gênero perante um conceito discriminatório e sexista pré-formulado pela sociedade. Ademais, vem de uma cultura cujo modelo é patriarcal, no qual o homem é o centro da razão, o ser dominador. Essa maneira androcêntrica de pensar ainda perpetua na sociedade contemporânea, sendo a mulher menosprezada e diminuída diante das relações sociais (Gomes, 2018; Brasil, 2020).

A Lei Maria da Penha estabeleceu uma real conquista por ser uma ferramenta extremamente importante para dar visibilidade ao fenômeno social, que é o assassinato de mulheres por circunstâncias de gênero. Visto que todo ser humano tem direito à vida, sendo sua proteção um imperativo Jurídico de Ordem Constitucional, conforme a Carta Magna de 1988, comumente conhecida como Constituição Federal. Por mais que a Lei Maria da Penha tenha criado mecanismos para coibir e prevenir a violência doméstica e familiar através de medidas de assistência e proteção às mulheres em situação de vulnerabilidade, o número de vítimas continua crescendo. Logo, não somente é indispensável conhecer as características do problema para coibir os assassinatos femininos, mas também é essencial programar ações efetivas de prevenção para a situação exequente em sociedade (Oliveira, et al., 2019; Paula, 2018; Meneghetti, et al., 2020).

No estado de Sergipe, como na maioria dos estados brasileiros, têm sido recorrentes casos de feminicídios. Desse modo, torna-se necessário delinear e dar a conhecer de forma abrangente, um quadro mais completo a respeito do assassinato de pessoas do sexo feminino, em razão de ser biologicamente pertencentes a essa condição e apresentar o contexto de medidas que possam levar ao melhor enfrentamento desta situação social adversa. Como efeito, torna-se necessário descrever e analisar as características do feminicídio, os tipos de agressões sofridas pelas mulheres, as formas de medidas protetivas, o papel da intervenção dos diferentes órgãos de segurança pública e a promoção à integralidade da saúde feminina no convívio social.

Em Sergipe os dados sobre feminicídio passaram a ser registrados a partir desde 2016. Os locais de dados ligados aos registros sobre o feminicídio tinham necessidade de serem explorados para se ter ideia sobre a abrangência interestadual dos casos de feminicídio e sua natureza e características, incluindo até o desfecho judicial. Por isso o foco da pesquisa foi estabelecer o perfil epidemiológico sobre o feminicídio no estado de Sergipe, no período de 2016 a 2019 e avaliar a distribuição espacial da mortalidade por feminicídio em mulheres no Estado de Sergipe.

\section{Metodologia}

Trata-se de um estudo de coorte de caráter descritivo e analítico com abordagem quantitativa, via coleta de dados secundários, sendo a população de estudo composta pela quantidade de mulheres vítimas de feminicídio. Todos os aspectos éticos preconizados pela Resolução: no 466/2012 do Conselho Nacional de Saúde e foi aprovado pelo Comitê de Ética em Pesquisa CAAE no: 28278719.4.0000.5371 (Parecer no 4.029.257.4).

O presente estudo reuniu todas as fontes de dados para prosseguimento do perfil do feminicídio em Sergipe com recorte temporal 2016 a 2019, particularizando as ocorrências desse crime nos 75 municípios do Estado de Sergipe. De acordo o Instituto Brasileiro de Geografia e Estatística (IBGE), o estado de Sergipe apresenta oito regiões geopolíticas: Alto Sertão, Baixo Sertão, Baixo São Francisco, Leste Sergipano, Agreste Central, Grande Aracaju (engloba a Região Metropolitana de Aracaju - RMA), Centro Sul e Sul Sergipano (Brasil, 2010).

A população específica compreendeu mulheres com idade acima de 18 anos, vítimas de feminicídio, assassinato por morte violenta e com inquérito instaurado determinando o crime, sendo excluídas aquelas cuja residência ou ocorrência não se deu no estado de Sergipe, e qualquer tipo de homicídio doloso contra mulheres que não fosse confirmado o feminicídio. 
Para realização da taxa de mortalidade foi utilizado dados do IBGE, dados estimados dos seus registros contabilizam total de 2.068.017 habitantes de ambos os sexos, com 789.760 indivíduos do sexo feminino, ponderando-se para a faixa etária de acima de 18 anos (Brasil, 2020).

Foram utilizados dados secundários do Departamento de Homicídios e Proteção à Pessoa (DHPP), Instituto Médico Legal de Sergipe (IML), Coordenadoria de Estatística e Análise Criminal (CEACRIM) da Secretaria de Segurança Pública de Sergipe (SSP/SE), Tribunal de Justiça de Sergipe (TJ/SE) e do Núcleo de Pesquisa da Secretaria de Segurança Pública de Sergipe (NAPSEC-SSP/SE). Os dados de mortalidade referem-se ao período de 2016 a 2019. Os dados suplementares foram trabalhados segundo a tipificação das mortes por feminicídio em todo Sergipe.

As informações apresentadas no trabalho são fundamentadas com base na literatura e em investigação científica realizada no IML, nos inquéritos instaurados e finalizados após decisão judicial no TJ, DHPP, CEACRIM, SSP e NAPSECSSP/SE. Consistiu em variáveis como a morte por feminicídio, dependendo da faixa etária, cor/raça, grau de escolaridade, agente causador da morte, local de ocorrência e situação ocupacional independente.

Os dados foram descritos por meio de frequência absoluta e relativa percentual. As variáveis categóricas foram descritas por meio de frequência absoluta e relativa percentual. As variáveis contínuas foram descritas por meio de média e desvio padrão. A hipótese de independência entre variáveis categóricas foi testada por meio do teste Qui-Quadrado de Pearson e Exato de Fisher. O nível de significância adotado foi de 5\% e o software utilizado foi o R Core Team 2020.

\section{Resultados}

A população estimada de mulheres, no estado de Sergipe, no período do estudo foi de $3.268,716$, entre as quais ocorreram 262 mortes violentas femininas e 70 foram a amostra de feminicídios analisados. Assim, dentro da quantidade de mortes da população feminina ocorrida no estado, houve uma elevação gradativa do número de feminicídios, com destaque para o ano de 2019 descritos na tabela 1.

A distribuição da ocorrência de feminicídios, na RMA e demais municípios do estado de Sergipe, acompanhada de importantes variáveis demográficas. Pode-se notar que entre as duas regiões não foi estatisticamente significante o número de ocorrências de feminicídio. A faixa etária de 18 a 44 anos teve predominância. Entretanto, a faixa etária de 45 a 60 anos teve destaque, na RMA, o aumento de feminicídio na idade dos 60 anos. Quanto ao agente causador da morte, em ambas as regiões, nota-se que o uso de armas de fogo sobrepujou todos os demais agentes. Para o local da ocorrência do feminicídio, predominou a residência das vítimas. Importante destacar que em todas as regiões as mulheres, sem atividade definida eram economicamente ativas. Foi estatisticamente significativa à ocorrência de feminicídio entre as mulheres de raça/cor parda. Pertinente ao grau de escolaridade das vítimas mereceu destaque, em todas as regiões, as dificuldades para obtenção de informações. Mesmo assim, foi constatado que o feminicídio ocorreu mais frequentemente entre as mulheres com grau de escolaridade fundamental incompleto. 
Tabela 1. Feminicídios na região metropolitana de Aracaju e nos demais municípios do estado de Sergipe e outras variáveis2016 a 2019.

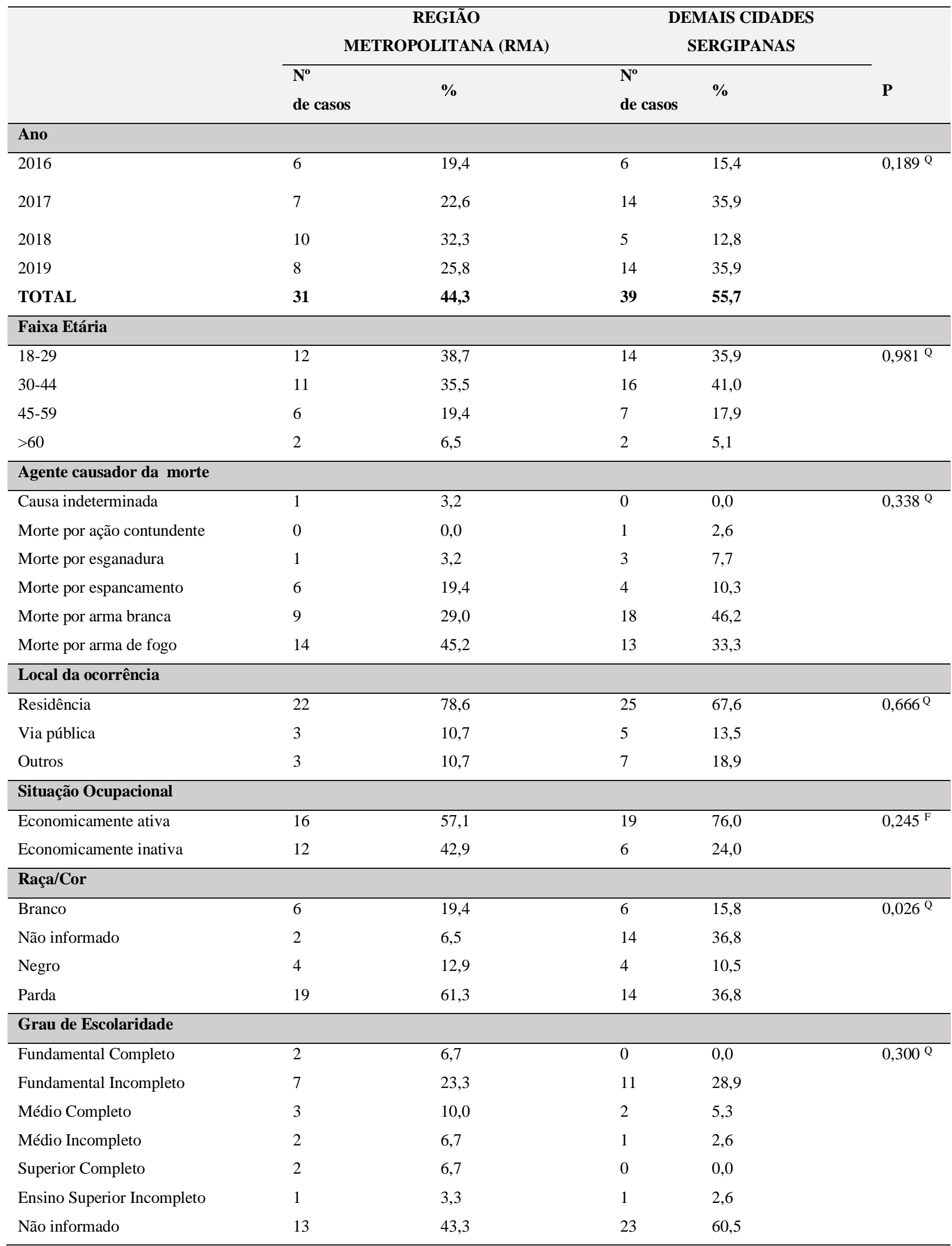

Q - Teste Qui-Quadrado de Pearson. F - Teste Exato de Fisher. Fonte de dados brutos: IML/CEACRIM. 
Quando observa-se a distribuição dos feminicídios por faixa etária durante o período do estudo, chama a atenção o valor absoluto de feminicídios na faixa de 45-59 anos de idade que foi 50\% do valor da faixa etária de 18-44 anos, em geral a mais frequente. Atenta-se, também, ao valor relativo que em 2019 chegou a 53,8\% dos 22 feminicídios ocorridos naquele ano (Tabela 2). As demais variáveis mostradas na tabela 2 não apresentaram significância estatística, apesar da predominância: do uso de armas de fogo; da ocorrência do feminicídio na residência; das mulheres economicamente ativas; da raça/cor parda; da escolaridade fundamental incompleta; e dos casos não informados quanto à escolaridade.

Tabela 2. Distribuição dos feminicídios e de outras variáveis, em todo estado de Sergipe, segundo a faixa etária - 2016 a 2019.

\begin{tabular}{|c|c|c|c|c|c|c|c|c|c|}
\hline & \multicolumn{8}{|c|}{ Faixa Etária } & \multirow[b]{3}{*}{$\mathbf{P}$} \\
\hline & \multicolumn{2}{|c|}{ 18-29 } & \multicolumn{2}{|c|}{$30-44$} & \multicolumn{2}{|c|}{$45-59$} & \multicolumn{2}{|r|}{$60+$} & \\
\hline & $\mathbf{N}^{\mathbf{o}}$ & $\%$ & $\mathbf{N}^{\mathbf{o}}$ & $\%$ & $\mathbf{N}^{\mathbf{o}}$ & $\%$ & $\mathbf{N}^{\mathbf{0}}$ & $\%$ & \\
\hline \multicolumn{10}{|l|}{ Ano } \\
\hline 2016 & 6 & 23,1 & 5 & 18,5 & 1 & 7,7 & 0 & 0,0 & 0,544 \\
\hline 2017 & 10 & 38,5 & 7 & 25,9 & 3 & 23,1 & 1 & 25,0 & \\
\hline 2018 & 4 & 15,4 & 7 & 25,9 & 2 & 15,4 & 2 & 50,0 & \\
\hline 2019 & 6 & 23,1 & 8 & 29,6 & 7 & 53,8 & 1 & 25,0 & \\
\hline \multicolumn{10}{|l|}{ Agente causador da morte } \\
\hline Causa indeterminada & 1 & 3,8 & 0 & 0,0 & 0 & 0,0 & 0 & 0,0 & 0,503 \\
\hline Morte p/ação Contundente & 1 & 3,8 & 0 & 0,0 & 0 & 0,0 & 0 & 0,0 & \\
\hline Morte por esganadura & 2 & 7,7 & 2 & 7,4 & 0 & 0,0 & 0 & 0,0 & \\
\hline Morte por espancamento & 2 & 7,7 & 4 & 14,8 & 3 & 23,1 & 1 & 25,0 & \\
\hline Morte por arma branca & 6 & 23,1 & 11 & 40,7 & 8 & 61,5 & 2 & 50,0 & \\
\hline Morte por arma de fogo & 14 & 53,8 & 10 & 37,0 & 2 & 15,4 & 1 & 25,0 & \\
\hline \multicolumn{10}{|l|}{ Local } \\
\hline Residência & 17 & 73,9 & 15 & 57,7 & 11 & 91,7 & 4 & 100,0 & 0,272 \\
\hline Via pública & 3 & 13,0 & 4 & 15,4 & 1 & 8,3 & 0 & 0,0 & \\
\hline Outros & 3 & 13,0 & 7 & 26,9 & 0 & 0,0 & 0 & 0,0 & \\
\hline \multicolumn{10}{|l|}{ Situação Ocupacional } \\
\hline Economicamente ativa & 16 & 76,2 & 13 & 68,4 & 6 & 54,5 & 0 & 0,0 & 0,133 \\
\hline Economicamente inativa & 5 & 23,8 & 6 & 31,6 & 5 & 45,5 & 2 & 100,0 & \\
\hline \multicolumn{10}{|l|}{ Raça/Cor } \\
\hline Branco & 6 & 24,0 & 3 & 11,1 & 2 & 15,4 & 1 & 25,0 & 0,438 \\
\hline Não informado & 7 & 28.0 & 6 & 22,2 & 1 & 7,7 & 2 & 50,0 & \\
\hline Negro & 2 & 8,0 & 4 & 14,8 & 1 & 7,7 & 1 & 25 & \\
\hline Parda & 10 & 40,0 & 14 & 51,9 & 9 & 69,2 & 0 & 0,0 & \\
\hline \multicolumn{10}{|l|}{ Escolaridade } \\
\hline Fundamental Completo & 0 & 0,0 & 1 & 3,7 & 1 & 7,7 & 0 & 0,0 & 0,266 \\
\hline Fundamental Incompleto & 9 & 37,5 & 7 & 25,9 & 2 & 15,4 & 0 & 0,0 & \\
\hline Médio Completo & 2 & 8,3 & 2 & 7,4 & 1 & 7,7 & 0 & 0,0 & \\
\hline Ensino Médio Incompleto & 3 & 12,5 & 0 & 0,0 & 0 & 0,0 & 0 & 0,0 & \\
\hline Ensino Superior Completo & 0 & 0,0 & 0 & 0,0 & 2 & 6,1 & 0 & 0,0 & \\
\hline Ensino Superior Incompleto & 0 & 0,0 & 0 & 0,0 & 1 & 3,0 & 1 & 6,3 & \\
\hline Não informado & 4 & 36,4 & 4 & 50 & 19 & 57,6 & 9 & 56,3 & \\
\hline
\end{tabular}

P - Teste Qui-Quadrado de Pearson. Fonte de dados brutos: IML/CEACRIM. 
A Tabela 3 apresenta a distribuição dos feminicídios na RMA e demais regiões do estado de Sergipe, segundo a raça/cor. Aparece bem caracterizada a significância estatística da presença da raça/cor preta e parda em todo o estado. Nota-se que ocorreu alguma dificuldade para obtenção desse tipo de informação, mas que não chegou a comprometer os achados. Fica demonstrada a frequência do uso da arma de fogo bem como a residência como o local de ocorrência do delito. A situação ocupacional revela que a raça/cor branca tem leve predomínio como economicamente ativa e aparece como menos inativa. Quanto à escolaridade os brancos possuíam um melhor percentual de ensino médio completo que as demais raças/cor.

Tabela 3. Feminicídios na região metropolitana de Aracaju e nos demais municípios do estado de Sergipe segundo a raça/cor 2016 a 2019

\begin{tabular}{|c|c|c|c|c|c|c|c|c|c|}
\hline & \multicolumn{8}{|c|}{ Raça/Cor } & \multirow[b]{3}{*}{$\mathbf{P}$} \\
\hline & \multicolumn{2}{|c|}{ Branco } & \multicolumn{2}{|c|}{ Negro } & \multicolumn{2}{|c|}{ Parda } & \multicolumn{2}{|c|}{$\begin{array}{c}\text { Não } \\
\text { Informado }\end{array}$} & \\
\hline & $\mathbf{N}^{\mathbf{0}}$ & $\%$ & $\mathbf{N}^{\mathbf{0}}$ & $\%$ & $\mathbf{N}^{\circ}$ & $\%$ & $\mathbf{N}^{\mathbf{0}}$ & $\%$ & \\
\hline \multicolumn{10}{|l|}{ Ano } \\
\hline 2016 & 2 & 16,7 & 1 & 12,5 & 7 & 21,2 & 2 & 12,5 & 0,074 \\
\hline 2017 & 2 & 16,7 & 3 & 37,5 & 5 & 15,2 & 10 & 62,5 & \\
\hline 2018 & 4 & 33,3 & 1 & 12,5 & 7 & 21,2 & 3 & 18,8 & \\
\hline 2019 & 4 & 33,3 & 3 & 37,5 & 14 & 42,4 & 1 & 6,3 & \\
\hline \multicolumn{10}{|l|}{ Agente causador da morte } \\
\hline Causa indeterminada & 0 & 0,0 & 1 & 12,5 & 0 & 0,0 & 0 & 0,0 & 0,138 \\
\hline Morte por ação contundente & 1 & 8,3 & 0 & 0,0 & 0 & 0,0 & 0 & 0,0 & \\
\hline Morte por esganadura & 1 & 8,3 & 0 & 0,0 & 3 & 9,1 & 0 & 0,0 & \\
\hline Morte por espancamento & 0 & 0,0 & 2 & 25,0 & 5 & 15,2 & 3 & 18,8 & \\
\hline Morte por arma branca & 3 & 25 & 4 & 50,0 & 13 & 39,4 & 6 & 37,5 & \\
\hline Morte por arma de fogo & 7 & 58,3 & 1 & 12,5 & 12 & 36,4 & 7 & 43,8 & \\
\hline \multicolumn{10}{|l|}{ Local } \\
\hline Residência & 7 & 63,6 & 6 & 75,0 & 23 & 74,2 & 11 & 73,3 & 0,73 \\
\hline Via pública & 1 & 9,1 & 0 & 0,0 & 5 & 16,1 & 2 & 13,3 & \\
\hline Outros & 3 & 27,3 & 2 & 25,0 & 3 & 9,7 & 2 & 13,3 & \\
\hline \multicolumn{10}{|l|}{ Situação Ocupacional } \\
\hline Economicamente ativa & 8 & 80,0 & 5 & 71,4 & 17 & 63,0 & 5 & 55,6 & 0,710 \\
\hline Economicamente inativa & 2 & 20,0 & 2 & 28,6 & 10 & 37,0 & 4 & 44,4 & \\
\hline \multicolumn{10}{|l|}{ Escolaridade } \\
\hline Fundamental Completo & 1 & 9,1 & 0 & 0,0 & 1 & 3,0 & 0 & 0,0 & 0,915 \\
\hline Fundamental Incompleto & 4 & 36,4 & 3 & 37,5 & 7 & 21,2 & 4 & 25 & \\
\hline Ensino Médio Completo & 2 & 18,2 & 0 & 0,0 & 2 & 6,1 & 1 & 6,3 & \\
\hline Ensino Médio Incompleto & 0 & 0,0 & 1 & 12,5 & 1 & 3,0 & 1 & 6,3 & \\
\hline Ensino Superior Completo & 0 & 0,0 & 0 & 0,0 & 2 & 6,1 & 0 & 0,0 & \\
\hline Ensino Superior Incompleto & 0 & 0,0 & 0 & 0,0 & 1 & 3,0 & 1 & 6,3 & \\
\hline
\end{tabular}

P - Teste Qui-Quadrado de Pearson. Fonte de dados brutos: IML/CEACRIM. 
Na tabela 4 pode ser notado que houve um predomínio de feminicídios ocorridos em via pública. Quanto à escolaridade com relação à situação ocupacional permaneceu predominante o ensino fundamental incompleto, um destaque para o percentual de ensino médio incompleto entre as mulheres de situação ocupacional incompleta.

Tabela 4. Feminicídios na região metropolitana de Aracaju e nos demais municípios do estado de Sergipe segundo a situação ocupacional - 2016 a 2019.

\begin{tabular}{|c|c|c|c|c|c|}
\hline & \multicolumn{4}{|c|}{ Situação ocupacional } & \multirow[b]{3}{*}{$\mathbf{P}$} \\
\hline & \multicolumn{2}{|c|}{ Ativa } & \multicolumn{2}{|c|}{ Inativa } & \\
\hline & $\mathbf{N}^{\circ}$ & $\%$ & $\mathbf{N}^{0}$ & $\%$ & \\
\hline \multicolumn{6}{|l|}{ Ano } \\
\hline 2016 & 7 & 20 & 3 & 16,7 & \multirow[t]{4}{*}{0,067} \\
\hline 2017 & 11 & 31,4 & 6 & 33,3 & \\
\hline 2018 & 4 & 11,4 & 7 & 38,9 & \\
\hline 2019 & 13 & 37,1 & 2 & 11,1 & \\
\hline \multicolumn{6}{|l|}{ Agente causador da morte } \\
\hline Causa indeterminada & 0 & 0,0 & 1 & 5,6 & \multirow[t]{6}{*}{0,095} \\
\hline Morte por ação contundente & 1 & 2,9 & 0 & 0 & \\
\hline Morte por esganadura & 3 & 8,6 & 1 & 5,6 & \\
\hline Morte por espancamento & 4 & 11,4 & 5 & 27,8 & \\
\hline Morte por arma branca & 10 & 28,6 & 8 & 44,4 & \\
\hline Morte por arma de fogo & 17 & 48,6 & 3 & 16,7 & \\
\hline \multicolumn{6}{|l|}{ Local } \\
\hline Residência & 5 & 15,2 & 1 & 6,3 & \multirow[t]{3}{*}{0,761} \\
\hline Via pública & 25 & 75,8 & 13 & 81,3 & \\
\hline Outros & 3 & 9,1 & 2 & 12,5 & \\
\hline \multicolumn{6}{|l|}{ Escolaridade } \\
\hline Ensino Fundamental Completo & 1 & 2,9 & 1 & 5,6 & \multirow[t]{7}{*}{0,224} \\
\hline Ensino Fundamental Incompleto & 11 & 32,4 & 5 & 27,8 & \\
\hline Ensino Médio Completo & 3 & 8,8 & 1 & 5,6 & \\
\hline Ensino Médio Incompleto & 0 & 0 & 3 & 16,7 & \\
\hline Ensino Superior Completo & 2 & 5,9 & 0 & 0 & \\
\hline Ensino Superior Incompleto & 2 & 5,9 & 0 & 0 & \\
\hline Não informado & 15 & 44,1 & 8 & 44,4 & \\
\hline
\end{tabular}

P - Teste Qui-Quadrado de Pearson. Fonte de dados brutos: IML/CEACRIM.

No TJS, durante o período do estudo, foram registrados 31 procedimentos investigatórios, todos em $1^{\circ}$ grau, demandados por Varas Criminais de 14 Comarcas de um total de 40 no estado de Sergipe. Esses processos cobriram os assuntos: homicídio qualificado, combinado à violência doméstica e familiar contra mulher e/ou feminicídio. Com este glossário pode-se observar que predominaram entre as categorias, a violência doméstica contra mulher com 16 casos, entre as 31 categorias. Como assunto, o destaque foi o feminicídio com 25 (80,6\%), entre as 31 tipificações de crimes. Este percentual de feminicídio representou 35,7\% dos 70 feminicídios, ocorridos durante o período do estudo. As medidas de proteção com base na Lei Maria da Penha tiveram fracas demanda. Foram apenas três solicitações, que em relação às 31 categorias de violência catalogadas, atingiram uma frequência de 9,67\%. Foram realizados 17 julgamentos, 12 sem resolução e cinco com resolução (Tabela 5). 
Tabela 5. Procedimentos processuais realizados pelo Tribunal de Justiça de Sergipe

\begin{tabular}{lll}
\hline & N & \\
\hline Categorias & & \\
\hline Violência doméstica contra mulher & 16 & 64,0 \\
Crime contra a vida & 11 & 44,4 \\
Crime comum & 2 & 8,0 \\
Crime contra a criança e adolescente & 2 & 8,0 \\
\hline Assuntos & & 100,0 \\
\hline Feminicídio & 25 & 4,00 \\
Homicídio simples & 1 & 12,0 \\
Homicídio qualificado & 3 & 8,0 \\
Crime contra a criança e adolescente & 2 & 68,0 \\
Julgamento/Decisão & 17 & 12,0 \\
Medidas protetivas de urgência & 3 & \\
\hline
\end{tabular}

Legenda: $\mathrm{n}$ - frequência absoluta. \% - frequência relativa percentual. Fonte: DIPLAD/TJS.

\section{Discussão}

O Atlas da Violência indicou que houve um crescimento dos homicídios femininos no Brasil, em 2017, com cerca de 13 assassinatos por dia. Ao todo, 4.936 mulheres foram mortas, o maior número registrado desde 2007. De acordo com o Monitor da Violência (2020), a população de mulheres no Brasil, no período de 2016-2019, era de 424.296,006. Neste período foram registrados 4.349 casos de feminicídio, os quais, por grupo de 100 mil mulheres, resultaram em taxas de 0,7, 1,0, 1,1, 1 e 1,2, respectivamente. No estado de Sergipe, nesse mesmo período, a população de mulheres era de 4.685,813 e ocorreram 46 feminicídios, cujos registros só tiveram início a partir de 2017, com taxas por grupo de 100 mil mulheres de 0,8, 1,4 e 1,8, respectivamente. O presente estudo, realizado no período de 2016-2019, com a inclusão dos dados referentes ao número de feminicídios do ano de 2016, compreendeu uma população estimada pelo IBGE de 3.268,716 mulheres acima de 18 anos, com residência no estado de Sergipe (Cerqueira, et al., 2020; Brasil, 2016).

Neste período, 262 mulheres acima de 18 anos sofreram morte violenta; entre essas mortes, 70 foram por feminicídio, o que significou uma taxa percentual geral de $26,7 \%$ casos de feminicídios por total de mortes dessas mulheres. As de feminicídio por grupo de 100 mil foram de 1,51, 2,60, 1,82, e 2,61, respectivamente. Entre as regiões geográficas brasileiras, a região CentroOeste lidera os resultados da pesquisa, aparecendo com uma taxa de 7,98, seguida pela Sudeste com 4,78, Norte com 4,77, Nordeste com 4,05 e a Sul com 3,82 mortes por feminicídio por 100 mil mulheres (Brasil, 2020; Cerqueira, et al., 2020).

Examinando especificamente os dados da Região Nordeste, não foram encontrados dados do ano de 2016 nos estados da Bahia, Ceará e Maranhão. Entretanto, em geral, as taxas de feminicídio de Sergipe se mantiveram mais semelhantes às do estado de Alagoas, que foram de (2,1, 1,9, 1,2 e 2,5 respectivamente). Mato Grosso do Sul, no período de 2016-2019 apresentou o maior número de feminicídio, 125, e as maiores taxas por grupo de 100 mil mulheres $(2,4,2,0,2,6$ e 2,1, respectivamente). (Monitor de violência, 2019)

Apesar das similaridades dos crimes de feminicídio, as taxas variam de acordo com o contexto sociocultural e político no qual as mulheres vivem (Sanz-Barbero, et al., 2016; Abrunhosa, et al., 2020). Toprak \& Ersoy (2017) destacaram que cada região, país e estado têm suas respectivas singularidades relacionadas ao delito praticado. A Turquia é um exemplo de país conservador que preza pela tradição, hierarquia e patriarcado. Analisado o feminicídio por região, nas oito regiões geopolíticas 
do estado de Sergipe, verificou-se que o número de feminicídio predominou na região geopolítica do grande Aracaju com quase $50 \%$ do total dos feminicídios ocorridos em todo o estado de Sergipe. Esta ocorrência pode estar relacionada, possivelmente, à concentração de indivíduos nessa região que, com 1.077, 132 habitantes, representa quase 50\% da população do estado.

As taxas de feminicídio variam de acordo com o contexto sociocultural e político no qual as mulheres vivem (Sanz-Barbero, et al., 2016; Silva, et al., 2013). Conforme Illicachi (2018), mulheres habitantes das zonas urbanas do Equador tinham maior probabilidade de sofrerem violência doméstica e serem vítimas de feminicídio em relacionamentos íntimos. Ilanzo, et al. (2018) também relataram que as mulheres que vivem em zonas rurais têm maior probabilidade de padecerem violência doméstica e serem vítimas de feminicídio em relacionamentos íntimos.

Pesquisa do Instituto de Pesquisa Econômica Aplicada (IPEA) relatou que a taxa de homicídios foi mais elevada em mulheres negras, contabilizando, de 2016 a 2018, 154 homicídios e entre as não negras, o número foi de 23 homicídios. Para o período de 2008 a 2018, 85\% das vítimas eram pretas e pardas, enquanto que 15\% eram brancas, amarelas ou indígenas. Só no ano de 2019 foram 1.326 vítimas de feminicídio no Brasil, das quais 66,6\% eram negras (Brasil, 2016). Do ponto de vista sociodemográfico o feminicídio tem sido traçado, em um perfil geral das vítimas, como: jovens, negras e com baixa escolaridade. O Instituto Sou da Paz, chamou atenção sobre a ausência de 18\% de registros sobre raça. De acordo com Oliveira, et al. (2019), independentemente da idade, as mulheres pardas ou negras são aquelas mais atingidas pelas violências, principalmente devido ao contexto histórico em que estão inseridas, que geralmente inclui condições precárias de moradia e estudo. No Brasil, as maiores vítimas do feminicídio são negras e jovens, com idade entre 18 e 30 anos. De acordo com os últimos dados do Mapa da Violência, a taxa de assassinato de mulheres negras aumentou 54\% em dez anos. O número de crimes contra mulheres brancas, em compensação, caiu $10 \%$ no mesmo período. No atual estudo foi significativa a ocorrência de feminicídio na raça negra e parda, bem como dos casos não informados.

Quanto à faixa etária, no presente estudo, prevaleceu sem significância estatística, a ocorrência de feminicídio, na faixa de 18-44 anos. Foram destaques o valor absoluto de feminicídios, em 2018, da faixa etária de 45-60, que chegou a 50\% do valor da faixa etária de 18-29 anos, bem como o aumento do valor relativo de feminicídios de 53,8\%, em relação ao total dos feminicídios ocorridos no ano de 2019. No Brasil, de acordo com o Mapa da Violência, as maiores vítimas do feminicídio são negras e jovens, com idade entre 18 e 30 anos. Para o IPEA 2016, as mulheres jovens foram as principais vítimas: 31\% estavam na faixa etária de 20 a 29 anos e $23 \%$ na de 30 a 39 anos, o que significa que mais da metade dos óbitos (54\%) foram de mulheres de 20 a 39 anos.

Montero (2019) destacou que as mulheres jovens e em idade reprodutiva são aquelas que possuíam maior probabilidade de fazerem parte das estatísticas feminicidas no México. Canevari \& Rosa (2016), em estudo realizado em Santiago del Estero (Argentina), destacaram que a média de idade das vítimas foi de 31,4 anos e que já vivenciaram algum tipo de violência desde as primeiras décadas de vida, sofrendo um aumento significativo ao atingirem 11 anos.

Um fato observado é que as maiores taxas de mortalidade ocorrem em mulheres jovens, sobretudo na segunda e terceira década de vida. Esses achados são corroborados por outros estudos desenvolvidos no Brasil, nos Estados Unidos, na Argentina, na Colômbia e no México. Essas informações indicam a necessidade de ações multissetoriais na construção de novas identidades contra a violência (Messias, 2020; Costa, 2020; Lobo, 2016).

Souza, et al. (2017), no tocante ao local onde o feminicídio ocorre, destaca que no Brasil nos últimos 35 anos houve distribuição semelhante entre domicílio (28,89\%), hospital (28,32\%) e via pública (26,58\%). O que não foi evidenciado nas regiões geográficas: no Sul, Norte e Centro-Oeste a maior proporção dos óbitos ocorreu no domicílio (37,56\%, 30,30\% e 33,82\%, respectivamente); seguido pelo hospital $(23,61 \%, 27,43 \%$ e 26,29\%, respectivamente). Em contrapartida, na região Nordeste a via pública foi o principal local de ocorrência dos óbitos (31,03\%), seguido pelo domicílio (27,58\%); na região Sudeste os principais locais de ocorrência do óbito foram o hospital (32,05\%), com menor percentual para a via pública (26,83\%) e o 
domicílio (26,04\%). Ainda, destacaram que nos quinquênios analisados verificou-se uma modificação entre o principal local de ocorrência do óbito. No atual estudo foi constatado que, no estado de Sergipe, o domicílio da vítima foi identificado como o lugar de maior ocorrência dos feminicídios em todas as cidades do estado. Em São Paulo, um levantamento realizado pelo Instituto Sou da Paz, com base nos números disponibilizados pela Secretaria de Segurança Pública do Estado e Corregedorias das Polícias Civil e Militar, relatou que, em 2019, para cada 10 vítimas de feminicídio, sete foram mortas dentro de casa. Na Colômbia, García \& Franco (2018) relataram que 41,46\% dos feminicídios ocorreram em domicílio.

Este contexto pode significar a presença de características socioeconômicas e de culturas regionais. Uma das ideias são aquelas situações nas quais a mulher tem menos poder ou menos recursos do que o homem. Outro fator diz respeito ao nível de escolaridade da mulher. O IPEA (2016) aponta entre essas situações: a maior parte das vítimas tinham baixa escolaridade, 48\% daquelas com 15 ou mais anos tinham até 8 anos de estudo e a vulnerabilidade econômica. O Instituto Sou da Paz considera que há influência econômica e afetiva no processo de violência, por meio do aumento considerável na mortalidade por feminicídio, relacionado a esse fator determinante. Os achados do presente estudo indicaram uma relação muito forte entre o feminicídio em Sergipe com as variáveis: local de ocorrência, raça/cor e escolaridade. Nesta última variável predominaram mulheres com ensino fundamental incompleto (Brasil, 2016).

O uso de armas de fogo prevaleceu em todos os cenários seguidos pelo uso de arma branca. Para o IPEA (2016), no Brasil, 50\% dos feminicídios envolveram o uso de armas de fogo e 34\%, de instrumento perfurante, cortante ou contundente. De acordo com Montero (2019), as formas empregadas para efetivação desse crime foram armas de fogo e branca, de maneira intencional ou não-intencional. Em todas as regiões brasileiras, entre 2016 e 2019, a agressão foi perpetrada em maior proporção por arma de fogo, seguida de objetos cortantes/contundentes e estrangulamento. Esses achados estão em consonância com a realidade evidenciada nos Estados Unidos da América e no Brasil (Souza, et al., 2017; Brasil, 2020).

Buscando dados sobre a demanda ao TJS constatamos apenas a instalação de 31 procedimentos investigatórios, todos em $1^{\circ}$ grau, que foram demandados por Varas Criminais de 14 Comarcas, do estado de Sergipe. No Portal do CNJ (2107), há um relato de que a região Nordeste é a que apresentou a menor demanda à Justiça, com média de 6,9 processos a cada mil mulheres residentes. Região Norte: 12,1 processos a cada mil mulheres; Região Sudeste: 12,4 processos a cada mil mulheres; Região Sul: 13,2 processos a cada mil mulheres residentes; Centro-Oeste: 19,3 processos a cada mil mulheres.

O Brasil ocupa o $5^{\circ}$ lugar no ranking mundial de Feminicídio, segundo o Alto Comissariado das Nações Unidas para os Direitos Humanos (ACNUDH). O país só perde para El Salvador, Colômbia, Guatemala e Rússia em número de casos de assassinato de mulheres. Em comparação com países desenvolvidos, aqui se mata 48 vezes mais mulheres que o Reino Unido, 24 vezes mais que a Dinamarca e 16 vezes mais que o Japão ou Escócia. Por aqui, muitos desses casos ocorrem em municípios de pequeno porte, onde não há delegacias da mulher. Dados do IBGE apontam que apenas 7,9\% dos municípios brasileiros têm delegacias especializadas para atender mulheres. Na ausência de uma delegacia especializada, as vítimas e seus familiares recorrem às delegacias tradicionais, onde há menos preparo dos policiais para lidar com casos desse tipo. Como consequência, pode aumentar a impunidade penal pelo não reconhecimento da violência baseada no gênero como crime (Brasil, 2020).

Juliana Gonçalves relata a frase de Bruna Pereira: "Violência no Brasil é um fenômeno social articulado a partir do racismo e do patriarcado". Diz ainda na página que escreveu: Mortes de mulheres no Brasil têm raça e classe definidas, dizem pesquisadores. Concordamos com esses pesquisadores, pelo que encontramos no presente estudo (Brasil de fato, 2020).

\section{Conclusão}

De acordo com os dados trazidos no estudo, o perfil epidemiológico do feminicídio encontrado em Sergipe teve como principal causa de mortalidade o fato de ser mulher, evidenciada dentro das oito regiões geopolíticas com distribuição semelhante dos casos tanto na RMA, quanto nos demais municípios. Esta realidade demonstra a elevada taxa de mortes violentas 
evidenciando a necessidade de discussão articulada que envolva políticas de saúde e ambiente, de sociedade e educação. Consiste em um problema de saúde pública, com consequências de magnitude sociais e culturais. É um fator multicausal que está associado a diversas condições que necessitam de maior atenção.

A taxa de feminicídio prevaleceu na RMA com alto número de mulheres acometidas no ano de 2019. Destacou-se, em termos de magnitude e prevalência de ocorrência dos casos de óbitos, que aconteceram na madrugada por arma de fogo na RMA e predominância da arma branca nas demais regiões do estado, tendo como vítima importante a mulher acima de 18 anos, parda, economicamente ativa e com ensino fundamental incompleto.

A pesquisa evidenciou, investigou e caracterizou as mulheres acometidas por esse tipo de crime, sendo a residência o local mais comum, constatado para ocorrência do homicídio. No conjunto das causas externas, a agressão destaca-se em termos de amplitude geral e, com predomínio de ocorrência dos casos, a mulher apresenta-se como vítima importante. Em suma, este é um fato evidenciado não apenas por dano social, mas por perdas na população economicamente ativa.

Com o intuito de contribuir para uma visão mais ampla no que concerne às atitudes e condições sociais de famílias e da sociedade, este estudo poderá colaborar com os gestores das esferas públicas para que sejam compreendidos quais os determinantes, fatores e consequências que levam a mortalidade de mulheres pelo feminicídio. Além disso, o conhecimento do perfil epidemiológico direciona o desenvolvimento de ações sociais de continuidade na comunidade, como medidas preventivas, protetivas, restaurativas e implementações de políticas públicas sociais.

Expressivas as evidências retratadas nesta pesquisa; se o panorama publicado sobre a mortalidade por feminicídio praticada contra mulheres não for revertido com brevidade, as expectativas futuras são preocupantes, em virtude das variadas formas de violência que tornam vítima ampla parcela da sociedade sergipana.

Modelos de políticas com base conceitual, construídos a partir de fatores atualizados, com dados que revelam a violência como tragédia no contexto social e familiar, devem ser implantados a partir de dados relevantes com o propósito de políticas públicas inclusivas e abrangentes, tendo como ponto de partida a análise deste estudo que revela a alta incidência de feminicídio, sendo reconhecido como um grupo vulnerável.

Para evitar fatores de risco de agressão às mulheres, é imprescindível que sejam adotadas políticas com medidas protetivas e programas que abordem os coeficientes de proteção. A partir disso, é essencial que essas práticas identifiquem os fatores que aumentam a suscetibilidade, visando o fortalecimento da rede de apoio, cuidado e proteção para o enfrentamento da violência contra pessoas do sexo feminino.

Ademais, essas ações poderão colaborar com os gestores das esferas públicas por meio da formulação de instrumentos que colaborem na identificação do crime de feminicídio. Dessa forma, conseguirão conhecer o perfil sociodemográfico do delito para desenvolver ações sociais de continuidade na comunidade.

Essa temática consiste em um problema de saúde pública, com consequências de magnitude sociais e culturais, é um fator multicausal que está associado a diversas condições que necessitam de maior atenção. Contudo, a articulação entre as redes de atenção que integram a promoção, prevenção e controle das mortes violentas femininas tornam-se imprescindíveis para minimizar o problema.

Outro ponto importante que pode ser observado no trabalho foi a busca pela análise do processo quantitativo dos dados realizado pelos sistemas de informações locais e nacionais de saúde e direito na área da interdisciplinaridade, pois permitem entender a abordagem do homicídio doloso qualificado de forma aprofundada. Com isso, servirá como direcionamento em relação às condutas de rastreamento das vítimas, e a detecção precoce com o auxílio da notificação compulsória com uso racional de recursos em saúde pública.

Sendo assim, tornou-se indispensável, descrever e analisar as características do feminicídio no estado de Sergipe, visto que, intensificam as perspectivas futuras dentro da Política de Atenção Integral a Saúde da Mulher de forma preventiva e 
resolutiva com medidas protetivas direcionadas e eficazes, e proporciona promoção de expectativa de vida dentro dessa população.

Em suma, a pesquisa possuiu exequibilidade e destinou-se no levantamento epidemiológico para formulação de dados estatísticos para avaliar de forma quantitativa a população feminina nordestina assassinada, e a característica da população com identificação dos fatores de risco, faixa etária, idade e local de ocorrência na abordagem das formas de vigilância ativa.

O feminicídio é apenas a ponta do iceberg da violência contra a mulher e representa o desfecho mais extremo do problema. As taxas de feminicídio variam de acordo com o contexto sociocultural e político no qual as mulheres A violência vem se alastrando, ano após ano, das mais diversas formas, nos nove estados da região Na região Nordeste, a questão da segurança pública se torna cada ano mais preocupante As mortes violentas por razões de gênero são um fenômeno global e vitimizam mulheres todos os dias, como consequência da posição de discriminação estrutural e da desigualdade de poder, que inferioriza e subordina as mulheres aos homens.

\section{Referências}

Abrunhosa, C., Rodrigues, A.D.C, Cruz, A.R., Gonçalves, R.A., \& Cunha, O. (2020). Crimes against women: from violence to homicide. Journal of interpersonal violence, 0886260520905547.

Brasil de fato. (2020). Uma visão popular do Brasil e do mundo. https://www.brasildefato.com.br/2017/11/07/mortes-de-mulheres-no-brasil- tem- raca-e-classedefinidas-dizem-pesquisadores

Brasil, A.B.S.P. (2018). Secretaria de Segurança Pública. Anuário Brasileiro de Segurança Pública, 2018.

Brasil, A.B.S.P. (2020). Secretaria de Segurança Pública. Anuário Brasileiro de Segurança Pública. Ford Open Society Foundations - OSF, 2020.

Brasil, I.B.G.E. (2016). Instituto Brasileiro de Geografia e Estatística-IBGE/CENSUS. Diretoria de Pesquisas, Coordenação de Trabalho e Rendimento. Censo Demográfico. Brasília, 2016.

Brasil, I.P.E.A. (2016). Ministério do Planejamento, Orçamento e Gestão. Instituto de Pesquisa Econômica Aplicada (IPEA). Brasília, 2016.

Brasil. (2010). Ministério da Saúde. DATASUS. Informações de saúde. Estatísticas de mortalidade: óbitos por ocorrência segundo causas externas do Brasil. Brasília, DF, 2010.

Canevari, C., \& Rosa, I.S.A.C. (2016). Territorios de violencia patriarcal: doce años de femicidios en Santiago del Estero (2002-2014). Trabajo y sociedad, (26), 257-284.

Cerqueira, D., Lima, R.S., Bueno, S., Neme, C., Ferreira, H., Coelho, D., Alves, P.P. Pinheiro, M.. Astolfi, R., \& Marques, D. Atlas da violência. Brasília/DF: IPEA, 2020.

Costa, F. (2020). Borderless A Study of Violence against Women in Universities: Brazil, Portugal, and the UK. Journal of International Women's Studies, 21(2), $169-177$.

García, J., \& Franco, J.A. (2018). El feminicidio en Bogotá, una mirada desde el abordaje médico-legal. Cuadernos de Medicina Forense, 24(1-2), 27-34.

Gomes, I.S. (2018). Feminicídios: um longo debate. Revista Estudos Feministas, 26(2).

Ilanzo, M.P., Urbano, O.M.C., Delgado, M.C., Ramirez, N. P., Mendoza, G. M. P., \& Garcia, A. E. O. (2018). Violencia extrema contra la mujer y feminicidio en el Perú. Revista Cubana de Salud Pública, 44, 278-294.

Illicachi, G.J. (2018). Las mujeres en un país plurinacional e intercultural, Ecuador. La ventana. Revista de estudios de género, v. 6, n. 48, p. 358-389, 2018.

Lobo, P.A. (2016). O feminicídio de juárez: alterações económicas, narrativas sociais e discursos coloniais na fronteira dos EUA e MÉXICO. Ex aequo, (34), 45-58.

Meneghel, S.N., Rosa, B.A.R.D., Ceccon, R.F., Hirakata, V.N., Danilevicz, I.M. (2017). Feminicídios: estudo em capitais e municípios brasileiros de grande porte populacional. Ciência \& Saúde Coletiva, 22, 2963-2970.

Meneghetti, B.P., Danilevicz, I.M., Polidoro, M., \& Plentz, L.M. (2020). Feminicídios em municípios de fronteira no Brasil. Rev Ciência \& Saúde Colet.

Messias, E.R., Carmo, V.M., \& Almeida, V.M. (2020). Femicide: an analysis from the perspective of the human person's dignity. Estudos Feministas, 28(1).

Monitor de violência. (2019). Feminicídio no Brasil. G1. https://especiais.g1.globo.com/monitor-da-violencia/2018/feminicidios-no-brasil/

Montero, C. (2019). Estudio comparado entre España y México sobre el marco jurídico aplicable al feminicidio. Boletín mexicano de derecho comparado, v. 52, n. 154, p. 147-170, 2019. 
Research, Society and Development, v. 10, n. 5, e47310515197, 2021

(CC BY 4.0) | ISSN 2525-3409 | DOI: http://dx.doi.org/10.33448/rsd-v10i5.15197

Oliveira, C.A.B.D., Alencar, L.N.D., Cardena, R.R., Moreira, K.F.A., Pereira, P.P.D.S., \& Fernandes, D.E.R. (2019). Perfil da vítima e características da violência contra a mulher no estado de Rondônia-Brasil. Revista Cuidarte, 10(1).

Paula, D.O.D. (2018). Human Rights and Violence Against Women: Campo Algodonero Case. Revista Estudos Feministas, 26(3).

Sanz-Barbero, B., Heras-Mosterio, J., Otero-García, L., \& Vives-Cases, C. (2016). Sociodemographic profile of femicide in Spain and its association with domestic abuse reporting. Gaceta sanitaria, 30(4), 272-278.

Sanz-Barbero, B., Otero-García, L., Boira, S., \& Marcuello, C. (2016). Femicide Across Europe COST Action, a transnational cooperation network for the study of and approach to femicide in Europe. Gaceta sanitaria, 30(5), 393-396.

Silva, C.D., Gomes, V.L.D.O., Acosta, D.F., Barlem, E.L.D., \& Fonseca, A.D. (2013). Epidemiologia da Violência Contra a Mulher: características do agressor e do ato violento. Journal of Nursing UFPE/Revista de Enfermagem UFPE, 7(1).

Souza, E.R.D., Meira, K.C., Ribeiro, A.P., Santos, J.D., Guimarães, R.M., Borges, L.F., ... \& Simões, T.C. (2017). Homicídios de mulheres nas distintas regiões brasileiras nos últimos 35 anos: análise do efeito da idade-período e coorte de nascimento. Ciência \& Saúde Coletiva, 22, $2949-2962$.

Toprak, S., \& Ersoy, G. (2017). Femicide in Turkey between 2000 and 2010. PloS one, 12(8).

World Health Organization (WHO). (2016). ONU: Taxa de feminicídios no Brasil é quinta maior do mundo; diretrizes nacionais buscam solução. https://nacoesunidas.org/onu-feminicidiobrasil-quinto-maior-mundo-diretrizes nacionais-buscam-solucao/

World Health Organization (WHO). (2015). United Nations. https://www.who.int/en 